\title{
Accurate assessment of antibiotic susceptibility and screening resistant strains of a bacterial population by linear gradient plate
}

\author{
LIU YuQing ${ }^{1}$, LI JingRan ${ }^{1,3}$, DU JiaFa ${ }^{1}$, HU Ming $^{1}$, BAI Hua ${ }^{1}$, QI Jing ${ }^{1}$, GAO Chao ${ }^{1,3}$, \\ WEI TianTian ${ }^{1,3}$, SU Hong ${ }^{1}$, JIN JianLing ${ }^{2}$ \& GAO PeiJi ${ }^{2 *}$ \\ ${ }^{1}$ Shandong Key Laboratory of Animal Diseases Control and Breeding, Institute of Animal Science and Veterinary Medicine, \\ Shandong Academy of Agricultural Science, Jinan 250100, China; \\ ${ }^{2}$ State Key Laboratory of Microbial Technology, Shandong University, Jinan 250100, China; \\ ${ }^{3}$ College of Animal Science, Qingdao Agricultural University, Qingdao 266109, China
}

Received December 31, 2010; accepted August 8, 2011

\begin{abstract}
The dynamics of a bacterial population exposed to the minimum inhibitory concentration (MIC) of an antibiotic is an important issue in pharmacological research. Therefore, a novel antibiotic susceptibility test is urgently needed that can both precisely determine the MIC and accurately select antibiotic-resistant strains from clinical bacterial populations. For this purpose, we developed a method based on Fick's laws of diffusion using agar plates containing a linear gradient of antibiotic. The gradient plate contained two layers. The bottom layer consisted of $15 \mathrm{~mL}$ agar containing the appropriate concentration of enrofloxacin and allowed to harden in the form of a wedge with the plate slanted such that the entire bottom was just covered. The upper layer consisted of $15 \mathrm{~mL}$ plain nutrient agar added with the plate held in the horizontal position. After allowing vertical diffusion of the drug from the bottom agar layer for $12 \mathrm{~h}$, the enrofloxacin concentration was diluted in proportion to the ratio of the agar layer thicknesses. The uniform linear concentration gradient was verified by measuring the enrofloxacin concentration on the agar surface. When heavy bacterial suspensions were spread on the agar surface and incubated for more than $12 \mathrm{~h}$, only resistant cells were able to form colonies beyond the boundary of confluent growth of susceptible cells. In this way, the true MIC of enrofloxacin was determined. The MICs obtained using this linear gradient plate were consistent with those obtained using conventional antibiotic susceptibility tests. Discrete colonies were then spread onto a gradient plate with higher antibiotic concentrations; the boundary line increased significantly, and gene mutations conferring resistance were identified. This new method enables the rapid identification of resistant strains in the bacterial population. Use of the linear gradient plate can easily identify the precise MIC and reveal the dynamic differentiation of bacteria near the MIC. This method allows the study of genetic and physiological characteristics of individual strains, and may be useful for early warning of antibiotic resistance that may occur after use of certain antimicrobial agents, and guide clinical treatment.
\end{abstract}

linear gradient plate, E. coli, enrofloxacin, MIC, resistant strain

Citation: Liu Y Q, Li J R, Du J F, et al. Accurate assessment of antibiotic susceptibility and screening resistant strains of a bacterial population by linear gradient plate. Sci China Life Sci, 2011, 54: 953-960, doi: 10.1007/s11427-011-4230-6

Determining the antibiotic susceptibility of pathogenic bacteria is an important function of clinical microbiology laboratories. For that reason, the Clinical and Laboratory

*Corresponding author (email: gaopj@sdu.edu.cn)
Standard Institute (CLSI) and similar organizations have established reference standards for antibiotic susceptibility testing (AST) methods and for determining pharmacokinetic $(\mathrm{PK}) /$ pharmacodynamics $(\mathrm{PD})$ breakpoints and clinical values. Using a standard testing method, we can determine the 
minimum inhibitory concentration (MIC) and inhibition zone diameter, which indicate whether the pathogen is sensitive or resistant to antibiotics used in clinical practice [1-3]. Over the years numerous countries around the world have been committed to the standardization of AST and surveillance of antibiotic-resistant bacteria [4-7]. However, the widespread use of antibiotics has significantly increased bacterial resistance [8-10], and the bacterial MIC far exceeds break point. For that reason, population dynamics of bacteria near the MIC is becoming an important question in the pharmacology of antibiotics. A novel method to precisely determine MICs is urgently needed, as well as the ability to identify "super" antibiotic-resistant strains in clinical bacterial populations.

As early as 1952, Szybalski [11] empirically established a gradient plate method to isolate antibiotic-resistant bacteria, but the method was used primarily to screen resistant strains rather than for AST [12-14]. In this study, we developed an improved method designed according to Fick's laws of diffusion $[11,15]$. A uniform linear increase in the antibiotic concentration along the gradient axis was verified by measuring enrofloxacin concentration on the agar surface. When heavy bacterial suspensions were spread on the agar surface and incubated for more than $12 \mathrm{~h}$, the boundary between resistant colonies and the confluent growth of susceptible cells demonstrated the true MIC of enrofloxacin and enabled identification of resistant strains. This method may be useful for early warning of antibiotic resistance to guide the clinical use of antibiotics.

\section{Materials and methods}

\subsection{Strain and materials}

Escherichia coli ATCC 25922, enrofloxacin, 1.5\% Luria Broth (LB) agar medium, 1.5\% agar medium, 9-cm petri dishes, 5-mL centrifuge tubes, phosphoric acid-triethylamine buffer, fluorescence spectrophotometer meter (HITACH F4500), 96-well plates.

\subsection{MIC determination of enrofloxacin to ATCC 25922 by microdilution method}

According to the CLSI standard [3], AST was performed with enrofloxacin using three dilution gradients: $1,0.5,0.25$, $0.125,0.062,0.031,0.016$, and $0.008 \mu \mathrm{g} \mathrm{mL}^{-1} ; 0.8,0.4,0.2$, $0.1,0.05,0.025,0.0125$, and $0.006 \mu \mathrm{g} \mathrm{mL}^{-1}$; and $0.6,0.3$, $0.15,0.075,0.038,0.019,0.009$, and $0.0045 \mu \mathrm{g} \mathrm{mL}^{-1}$.

\subsection{Determination of enrofloxacin MIC against $E$. coli ATCC 25922 by agar dilution method}

Enrofloxacin concentration was set as the dilution gradient of $1,0.5,0.25,0.125,0.062,0.031,0.016,0.008 \mu \mathrm{g} \mathrm{mL}^{-1}$, according to the CLSI standard. For AST, the bacteria were spread onto the plate at $10^{8}$ or $10^{3} \mathrm{CFU}$, instead of point inoculation [3].

\subsection{Enrofloxacin concentration-killing curve (CKC) for $E$. coli ATCC 25922}

LB agar plates were prepared with a linear gradient of 0.01 , $0.015,0.02,0.025,0.03,0.035,0.04,0.045,0.05 \mu \mathrm{g} \mathrm{mL}{ }^{-1}$ enrofloxacin. About 1000 bacterial cells were spread evenly on each plate and incubated at $37^{\circ} \mathrm{C}$ for $24 \mathrm{~h}$. The number of colonies in each plate was then counted, and the CKC equation was fitted to the data (GraphPad Prism 4.0 software): $N=\frac{N_{0}}{1+e^{r\left(x-B C_{50}\right)}}$, in which $N_{0}$ is the number of bacteria inoculated per plate, $N$ is number of colonies, $x$ is the drug concentration, $B C_{50}$ is the median bactericidal concentration, and the constant $r=-\frac{\text { slope }}{N_{0} / 4}$ is determined by the slope of the curve at $B C_{50}$ and $N_{0}[16]$.

\subsection{Determination of enrofloxacin recovery rate in the agar}

A standard curve between fluorescence intensity and enrofloxacin concentration was constructed. A dilution series of $0.1,0.01,0.001,0.0001,0.00001 \mu \mathrm{g} \mathrm{mL}^{-1}$ enrofloxacin solution was prepared with phosphoric acid-triethylamine buffer (pH 3.0). Fluorescence intensity was detected with a F4500 fluorescence spectrophotometer (excitation wavelength $278 \mathrm{~nm}$, absorption at $446 \mathrm{~nm}$, voltage $600 \mathrm{~V}$ ) [17].

A medium containing $1.5 \%$ agar and $1,2,4$, or $8 \mu \mathrm{g}$ $\mathrm{mL}^{-1}$ enrofloxacin was poured into $9-\mathrm{cm}$ petri dishes $(15$ $\mathrm{mL} / \mathrm{dish})$. After the agar solidified, $10-\mathrm{mm}$ circular agar blocks were removed. Each block was weighed, placed into $1 \mathrm{~mL}$ phosphate-triethylamine buffer $(\mathrm{pH} 3.0)$ in a $5-\mathrm{mL}$ centrifuge tube, and heated in water bath to dissolve the agar, and then cooled. The heating and cooling steps were carried out without agitation to allow the agar to settle. The supernatant was used to measure fluorescence intensity of enrofloxacin, and the concentration of each block was determined using the standard curve.

\subsection{Preparation and verification of a linear gradient plate}

Two layers of agar were poured into each of the 24 linear gradient plates. A bottom layer consisting of $15 \mathrm{~mL} \mathrm{LB}$ agar (about $55^{\circ} \mathrm{C}$ ) with $2 \mu \mathrm{g} \mathrm{mL} \mathrm{m}^{-1}$ enrofloxacin was allowed to harden in the form of a wedge with the plate slanted such that the entire bottom was just covered. The tilt direction was marked with an arrow. With the plate in the normal horizontal position, another $15 \mathrm{~mL}$ plain LB agar was added. After allowing vertical diffusion of the drug from the bot- 
tom agar surface for $12 \mathrm{~h}$, the enrofloxacin concentration was diluted in proportion to the ratio of the agar layer thicknesses. In this way, the enrofloxacin gradually increased from the theoretical concentration of 0 to $2 \mu \mathrm{g} \mathrm{mL}{ }^{-1}$ along the gradient axis (Figure 1A).
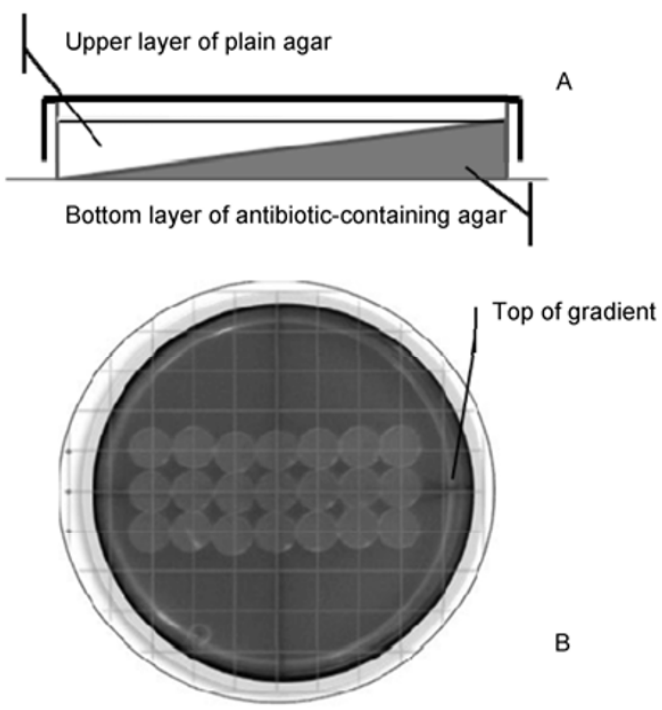

Figure 1 Gradient plate consisting of two agar layers.

LB medium with $1.5 \%$ agar $(15 \mathrm{~mL})$ was poured into 9-cm petri dishes and solidified. Then $10-\mathrm{mm}$ circular agar blocks were removed, weighed, and affixed to the solidified linear gradient plate surface equidistant along the tilt axis in three rows with seven blocks per row (Figure 1B). The 24 gradient plates were then incubated at $37^{\circ} \mathrm{C}$. Every hour, all the blocks on one of the plates were removed, and each block was placed into $1 \mathrm{~mL}$ phosphoric acid-triethylamine buffer ( $\mathrm{pH} 3.0$ ) in a 5-mL centrifuge tube to determine the enrofloxacin concentration.

1.7 Determination of enrofloxacin MIC against $E$. coli ATCC 25922 using the gradient plate

Overnight cultures of E. coli ATCC $25922\left(100 \mu \mathrm{L}, 10^{9}\right.$ CFU mL ${ }^{-1}$ ) were spread evenly onto the gradient plate (top concentrations of $0.01,0.02,0.04,0.08$, and $0.1 \mu \mathrm{g} \mathrm{mL}^{-1}$ ) and incubated at $37^{\circ} \mathrm{C}$ for $12 \mathrm{~h}$. Resistant cells forming col- onies beyond the boundary of confluent growth indicated the true enrofloxacin MIC against the bacterial population. The plate in which the natural boundary appeared in the middle was selected. The distance from the lowest concentration point to the boundary along the tilt axis was measured. MIC was calculated according to the linear gradient relationship and antibiotic concentration.

\subsection{Characteristics of the inoculum spread on the gra- dient plate}

All the discrete colonies (large and small) and some samples of bacteria from the area of dense confluent growth were inoculated into antibiotic-free liquid medium, incubated for $12 \mathrm{~h}$, and then spread onto a higher concentration gradient plate. Bacterial growth and colony location were then analyzed. In addition, the quinolone resistance-determining regions of gyrA, gyrB, and parC, parE were amplified from the total DNA of each colony, and mutations related to drug-resistance were analyzed (Table 1) [18].

\section{Results}

\subsection{Determination of enrofloxacin MIC against $E$. coli ATCC 25922 using the microdilution method}

As indicated in Figure 2, the three different gradients of double-dilution enrofloxacin produced three different MIC results by the microdilution method. The finding $0.025 \mu \mathrm{g}$ $\mathrm{mL}^{-1}$ was most accurate, but was obtained using an artificially set gradient. When the tested strain possesses high resistance, such artificially set gradient will produce greater errors in the estimated MIC. In addition, this method cannot differentiate resistant strains from the susceptible strains in the bacterial population.

\subsection{Determination of enrofloxacin MIC against $E$. coli ATCC 25922 using the agar dilution method}

This method also uses an artificially set gradient. However, the drug-resistant strains can be isolated using the plates prepared with specific antibiotic concentrations. As shown in Figure 3, the MIC was $0.0625 \mu \mathrm{g} \mathrm{mL}^{-1}$ when $1200 \mathrm{CFU}$

Table 1 PCR primer sequences for $g y r A, \operatorname{gyr} B$, parC, and parE

\begin{tabular}{|c|c|c|c|}
\hline Target genes & Primer sequences $\left(5^{\prime}\right.$ to $\left.3^{\prime}\right)$ & Primer length (bp) & Amplicon length (bp) \\
\hline \multirow{2}{*}{ gyrA } & F: TGCCAGATGTCCGAGAT & 17 & \multirow{2}{*}{269} \\
\hline & R: GTATAACGCATTGCCGC & 17 & \\
\hline \multirow{2}{*}{ gyrB } & F: GCCTTTCTTCACTTTGTACAGCG & 23 & \multirow{2}{*}{269} \\
\hline & R: GTGACGGCGGTACTCACCTG & 20 & \\
\hline \multirow{2}{*}{$\operatorname{parC}$} & F: TATGCGATGTCTGAAC & 16 & \multirow{2}{*}{264} \\
\hline & R: GCTCAATAGCAGCTCGGAAT & 20 & \\
\hline parE & F: CTGACCGAAAGCTACGTCAACC & 22 & 264 \\
\hline
\end{tabular}




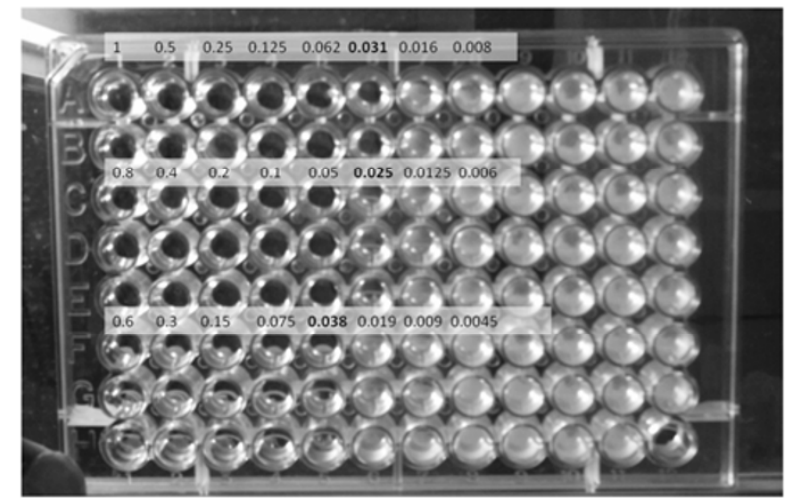

Figure 2 Enrofloxacin MICs against E. coli ATCC 25922 were 0.031, $0.025,0.038 \mu \mathrm{g} \mathrm{mL}^{-1}$ as assessed by the microdilution method.

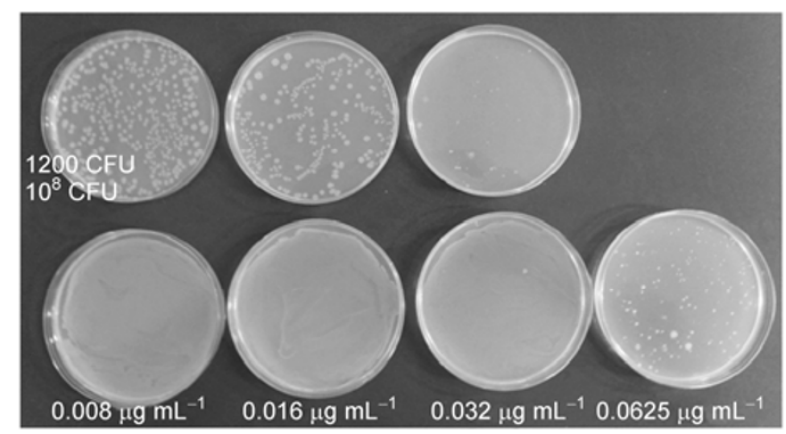

Figure 3 Enrofloxacin MICs against E. coli ATCC 25922 were 0.0625 and $0.125 \mu \mathrm{g} \mathrm{mL}^{-1}$ as assessed by the agar dilution method.

inoculum was used, but increased to $0.125 \mu \mathrm{g} \mathrm{mL} \mathrm{m}^{-1}$ with $10^{8}$ CFU. The problems associated with the two common AST methods recommended by CLSI were discussed in detail in our review [19].

\subsection{Determination of enrofloxacin MIC against $E$. coli ATCC 25922 using the CKC method}

The bactericidal effect according to enrofloxacin concentration was fully described by the CKC (Figure 4), with $B C_{50}=0.0205 \mu \mathrm{g} \mathrm{mL}^{-1}$ and $B C_{1}=0.0364 \mu \mathrm{g} \mathrm{mL}^{-1}$. Fitting the data eliminated the error introduced by the artificial gradient. However, the gradient was stepwise, not linear. Discrete colonies were obtained only between $B C_{1}$ and $B C_{50}$. We can rule out the emergence of mutant colonies, because inoculums were less than $1000 \mathrm{CFU}$ to facilitate colony counting [17].

\subsection{Determination and verification of linear enrofloxa- cin concentration on the gradient plate surface}

The standard curve of enrofloxacin concentration and fluorescence intensity, $y=52666 x+306.2$, showed a strong linear correlation $\left(R^{2}=0.998\right)$. The recovery rate of enrofloxacin from agar blocks was $99 \%$, indicating that the agar $(1.5 \%$, w/v) did not absorb significant amounts of enrofloxacin.

The time-concentration curves in Figure 5 show that enrofloxacin concentrations in different regions of the gradient plate surface increased rapidly over time. The higher enrofloxacin concentration in the bottom agar layer diffused more rapidly. After $12 \mathrm{~h}$, the enrofloxacin concentration at each point stabilized, and concentration-distance curves could be fitted with a linear equation (Figure 6). In this way, the drug concentration at any point on the agar surface along the gradient could be accurately determined.

\subsection{Determination of enrofloxacin MIC against $E$. coli ATCC 25922 using the gradient plate}

In the gradient plate prepared with $0.03125 \mu \mathrm{g} \mathrm{mL}{ }^{-1}$ enrofloxacin (bottom agar layer), two distinct regions were observed: an area of continuous dense growth and an area of discrete colonies (Figure 7A). The boundary concentration was $\mathrm{MIC}=0.018 \mu \mathrm{g} \mathrm{mL}^{-1}$. The gradient plate thus displayed continuous changes of the population regarding colony formation and regional distribution under increasing drug concentrations. This technique is a simple and intuitive method to visualize the CKC. Furthermore, it can identify

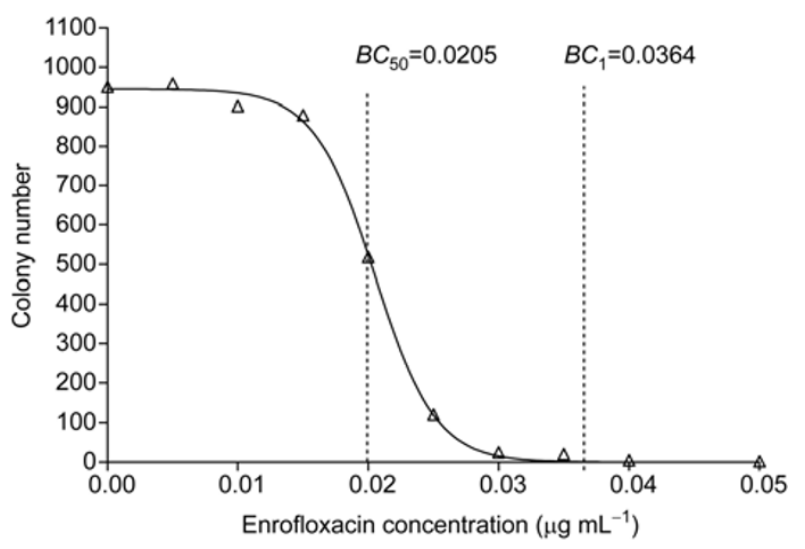

Figure 4 Enrofloxacin CKC against E. coli ATCC 25922 using the CFU counting method.

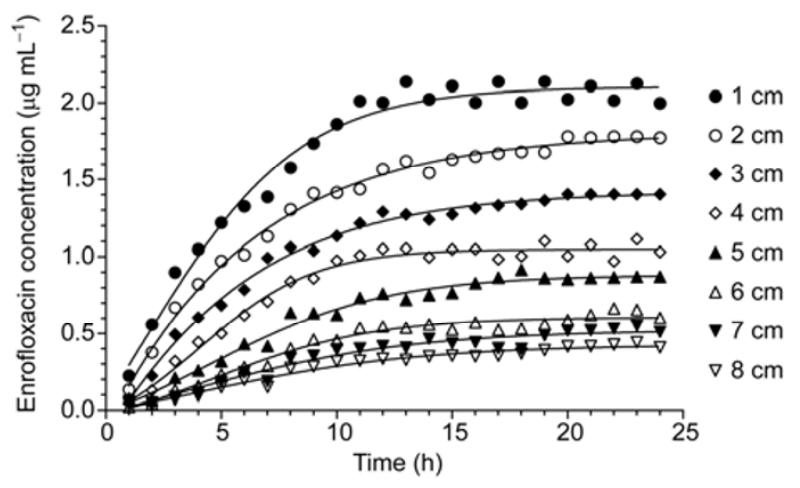

Figure 5 Enrofloxacin concentrations at different times in various locations along the gradient. The legend indicates the distance between the test point and the point of highest concentration. 


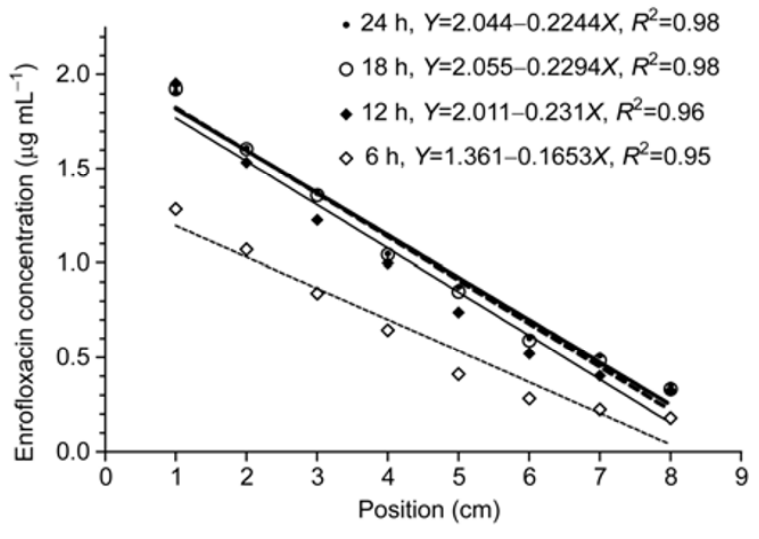

Figure 6 Enrofloxacin concentrations in various locations at different times fitted to linear equations.

resistant strains in the bacterial population for further genetic and physiologic analysis.

With increasing inoculum amounts, the number of discrete large colonies increased, but the location of the MIC line was unaltered (data not shown).

\subsection{Characteristics of the inoculum spread on the gra- dient plate}

Bacteria from three different locations of the continuous growth area were sampled, and three discrete large colonies and three discrete small colonies (Figure 7A) were also selected for analysis. These samples were inoculated separately into liquid LB medium, incubated for $8 \mathrm{~h}$, and then spread onto new gradient plates with different antibiotic concentrations.

Figure 7B shows that when bacteria from the continuous dense area were spread onto the same concentration gradient plate, the distribution of the colonies was consistent with that of Figure $7 \mathrm{~A}$, indicating that the characteristics of the susceptible bacteria were not influenced by the drug. Resistant strains in the original population, which were inhibited by the more numerous susceptible cells in the areas of continuous dense growth, formed large discrete colonies in the areas of higher antibiotic concentration.

Bacteria from the small colonies were then spread onto gradient plates prepared with $0.125 \mu \mathrm{g} \mathrm{mL}^{-1}$ enrofloxacin. The colony distribution pattern was also consistent with Figure 7A, but the boundary concentration was four times higher (Figure 7C). Spreading the large discrete colonies on plates prepared with $0.125 \mu \mathrm{g} \mathrm{mL}^{-1}$ enrofloxacin produced a similar pattern; however, the boundary concentration was 16 times higher (Figure 7D).

None of the colonies analyzed showed gene mutations in gyrB, parC, or parE. However, mutations were found in amino acid 87 of gyrA: GGC $\rightarrow$ TAC, glycine $\rightarrow$ tyrosine (small discrete colonies); GGC $\rightarrow$ TAC, glycine $\rightarrow$ tyrosine (two large discrete colonies); and GGC $\rightarrow$ GAC, glycine $\rightarrow$
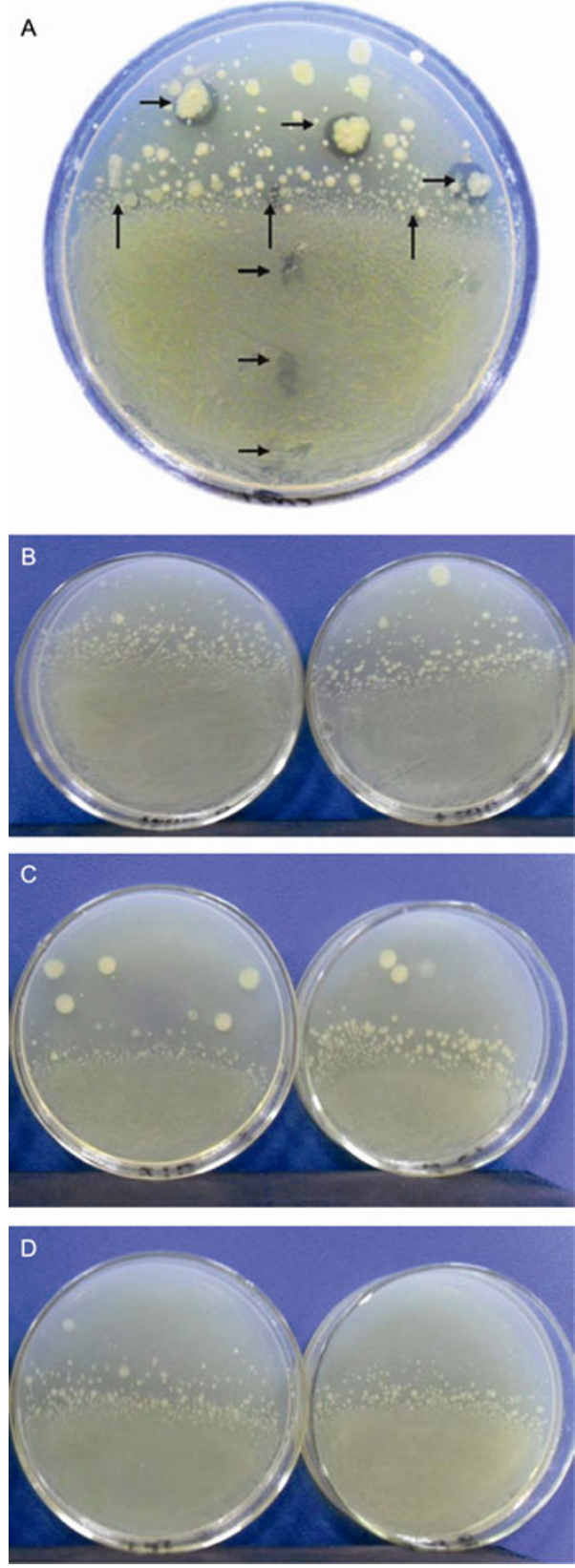

Figure 7 A, Area of smooth continuous dense growth and small colonies, and area of large discrete colonies of E. coli ATCC 25922 on the gradient plate prepared with $0.03125 \mu \mathrm{g} \mathrm{mL}^{-1}$ enrofloxacin. Arrows show colonies chosen for further analysis. B, Distribution of colonies after spreading bacteria from different locations of the continuous dense growth shown in Figure 7A onto a gradient plate prepared with $0.03125 \mu \mathrm{g} \mathrm{mL} \mathrm{m}^{-1}$ enrofloxacin. C, Distribution of colonies after spreading bacteria from three small discrete colonies shown in Figure 7A onto a gradient plate prepared with $0.125 \mu \mathrm{g} \mathrm{mL} \mathrm{L}^{-1}$ enrofloxacin. D, Distribution of colonies after spreading bacteria from three large discrete colonies shown in Figure 7A onto a gradient plate prepared with $0.5 \mu \mathrm{g} \mathrm{mL} \mathrm{m}^{-1}$ enrofloxacin.

aspartic acid (one large discrete colony).

\section{Discussion}

Problems with current AST methods and standards pro- 
posed by CLSI were previously discussed [19]. The disk diffusion method recommended by the World Health Organization is easy and has been widely used for nearly 40 years [2-4]. The E-test and spiral gradient endpoint method have been used for more than 20 years, but are still expensive because the technology is protected by patent [20,21]. In both methods, the drug diffuses into the agar plate in a three-dimensional hemispherical manner. The non-linear gradient diffusion and different physical and chemical properties of drug molecules are difficult to fit with a universal model [22-26]. The antibiotic concentration at the edge of the inhibition zone cannot be used to determine MIC, and using the diameter is also a poor indicator of MIC; therefore, MIC is rarely reported using these methods. The Kirby-Bauer method was established to determine the linear relationship between the inhibition zone diameter and MIC. However, there is not a one-to-one correspondence; when testing a large number of strains, one inhibition zone diameter may correspond to more than one MIC, and vice versa.

Because testing conditions (e.g., drug disk size drug dosage, bacterial inoculum, agar concentration, incubation time, and temperature) affect formation of the inhibition zone, improvement of the agar dilution method has been discussed for many years [27-32].

It is difficult to produce a continuous linear gradient using the agar dilution method; the measured concentration is a range of concentrations. The same problem exists when determining the mutation prevention concentration (MPC) for single-step mutants [33]. The CKC method was proposed to describe dynamic changes in bacterial populations exposed to linear concentration increases of a drug. The CKC from $B C_{99}$ to $B C_{1}$ can be used to calculate the $B C_{50}$ and other parameters $[17,34,35]$. The differential analysis method is based on turbidity to exclude the interference of resting cells in the bacterial population. This technique can be combined with a two-dimensional contour map to show the effects of drug concentration and time [36]. Both methods can provide important information for PK/PD analysis [37,38].

These conventional methods have the same problem in that they cannot directly determine the precise MIC, but produce mixed populations or colonies at the concentration near the MIC. It is impossible to isolate strains that are resistant to concentrations just above the MIC. However, use of linear gradient agar plates solves these difficulties.

Fick's laws of diffusion characterize the one-dimensional diffusion of small molecules in a homogeneous liquid phase. This is expressed as $\frac{\mathrm{d} C}{\mathrm{~d} t}=D \frac{\mathrm{d}^{2} C}{\mathrm{~d} x^{2}}$, in which $D$ is the diffusion coefficient, $C$ is the solute concentration, $x$ is diffusion displacement, and $t$ is diffusion time $[15,39]$. The drug concentration at all points in the bottom agar layer $C$ is the same before the upper agar layer is added. After the upper layer is poured, the molecules diffuse in the direction perpendicular to the slope of the bottom agar layer. Over time, $\frac{\mathrm{d}^{2} C}{\mathrm{~d} x^{2}}$ decreases rapidly, approaching zero as $t$ approaches infinity, while the constant $\frac{\mathrm{d} C}{\mathrm{~d} x}$ is greater than 0 , thus a continuous linear gradient forms in the direction perpendicular to the slope of the bottom agar layer. Because the upper agar surface intersects with the surface of the bottom agar layer, the concentration on the upper surface forms a linear gradient. This is the theoretical principle of the gradient plate.

In this paper, LB agar plates containing linear gradients of enrofloxacin were prepared. The diffusion equilibrium was approached at $12 \mathrm{~h}$, and the stable linear gradient was verified. When a large amount of bacteria was grown on the gradient plates, two types of colonies were observed in two different areas of the plate, thereby allowing the accurate determination of enrofloxacin MIC. Two types of mutant strains were isolated from the discrete colonies.

The linear gradient plate method provides significant advantages and shows potential for use in antibiotic resistance research and clinical AST.

(i) Accurate determination of the MIC. The bacterial population naturally forms two regions on the gradient plate. The boundary between the two regions provides an accurate assessment of the MIC. This method is superior to the artificial dilution series in tubes or the agar dilution method, particularly when evaluating high-resistant strains. The linear gradient plate and CKC methods are different approaches that provide equally satisfactory estimates of the bactericidal effect and resistance of the population exposed to a continuous linear gradient of enrofloxacin. The $B C_{50}$ $\left(0.0205 \mu \mathrm{g} \mathrm{mL}^{-1}\right)$ and boundary concentration $(0.018 \mu \mathrm{g}$ $\left.\mathrm{mL}^{-1}\right)$ were very close and were equivalent to the smallest MIC $\left(0.025 \mu \mathrm{g} \mathrm{mL}^{-1}\right)$ of the microdilution assay. However, of the three results, the concentration at the natural boundary $\left(0.018 \mathrm{~g} \mathrm{~mL}^{-1}\right)$ is a more accurate MIC of the dominant population. For example, when the bacterial inoculum increased (Figure 3), the inhibitory concentration appeared to increase, as assessed by the number of colonies in the agar dilution method. We were unable to determine the CKC (Figure 4) since the bacterial growth was dense, and the colonies too small to count. In contrast, the linear gradient plate reveals the precise MIC of dominant population stably.

(ii) Analysis of bacterial resistance. The heterogeneity of drug sensitivity displayed when the bacterial population increased reflects the emergence of strains with different resistance mutations and phenotypes. These discrete colonies from the region of high antibiotic concentration were spread on a new gradient plate, and a new MIC was obtained (the MPC). The mechanisms of resistance revealed 
the diversity of strains in the population, and resistance phenotypes were not limited to one-step mutations. The gradient plate method thus provides a new method for studying bacterial resistance phenotypes.

(iii) Early warning of drug-resistant strains may be used to determine clinical treatment. The gradient plate method can quickly identify consecutive drug-resistant strains. This method can be used to study the increase of resistant phenotypes, mutation rates, biochemical mechanisms of resistance mechanisms, and their molecular regulation.

(iv) The linear gradient plate can show individual bacteria changes of colony proliferation, reveal growth inhibition and death according to drug concentration and time, and evaluate time dependence or concentration dependence of the drug.

Based on differential equations and the contour map, we have proposed a new method to characterize the combined roles of drug concentration and treatment time. This method is suitable for laboratory studies to determine the mechanism of action and for PK/PD analysis [36]. The linear gradient plate method is simple and feasible for use in a typical clinical laboratory. The combination of the two methods enables a more comprehensive understanding of interactions between drugs and bacterial populations. However, its universality, availability, and potential clinical applications require further testing and improvement.

We thank Zhao Yue (State Key Laboratory of Microbial Technology, Shandong University) for performing the assays and Guo Lin (School of Mathematics and Information Science, Shandong Institute of Business and Technology) for the differential equation derivation. This work was supported by the National Natural Science Foundation of China (Grant No. 81171621), Shandong Province Young and Middle-Aged Scientists Research Awards Fund (Grant No. 2006BS02008), and China Postdoctoral Science Foundation Funded Project (Grant No. 20080440451).

1 Wheat P F. History and development of antimicrobial susceptibility testing methodology. J Antimicrob Chemother, 2001, 48: 1-4

2 Bauer A W, Kirby W M, Sherris J C, et al. Antibiotic susceptibility testing by a standardized single disk method. Am J Clin Pathol, 1966, 45: 493-496

3 CLSI. Performance Standards for Antimicrobial Susceptibility Testing. Twentieth. Informational Supplement. CLSI Document M100-S20. Wayne: Clinical and Laboratory Standard Institute, 2010

4 World Health Organization. Standardization of Methods for Conducting Microbiology Sensitivity Tests. Second Report of the Expert Committee on Antibiotics. WHO Technical Report Series, No. 210. WHO, Geneva, 1961

5 Gould I M. Towards a common susceptibility testing method? J Antimicrob Chemother, 2000, 45: 757-762

6 Yang Q W, Wang H, Xu Y C, et al. Introduction current situation and research of antimicrobial resistance surveillance in teaching hospitals of China. Chin J Clin Pharm, 2008, 24: 570-573

7 Xiao Y H. Introduction of Mohnarin (Ministry of Health National Antimicrobial Resistance Investigation Net. Chin J Antibiotics, 2008, 33: $577-588$

8 Taubes G. The Bacteria fight back. Science, 2008, 18: 356-361

9 Richard G W, Lewis K, Salyers A A, et al. Bacterial Resistance to Antimicrobials. 2nd ed. Boca Raton: CRC Press, 2007

10 Zhu X L, Qi J, Bai H, et al. Multi-drug resistance and hereditability of Escherichia coli isolated from animals in Shandong. Chin J Health Labor Tech, 2009, 19: 1473-1476

11 Bryson V, Szybalski W. Microbial selection. Science, 1952, 116: $45-51$

12 Shimkets L J, Dworkin M, Keller K H. A method for establishing stable concentration gradients in agar suitable for studying chemotaxis on a solid surface. Can J Microbiol, 1979, 25: 1460-1467

13 Zhang J E, Zhu J P, Liu Y X, et al. Screening of high gibberellin-producing strain from chloramphenicol resistant mutants. Chin J Antibiotics, 2005, 30: 301-303

14 Chen S Y, Deng Z X. Development of transformation system for Strepomyces hygroscopicus var Jingganshan JG5008. Chin J Appl Environ Biol, 2000, 6: 267-270

15 Nelson P (author), Li M, Dai L R (translation). Biological Physics: Energy, Information and Life. Shanghai: Shanghai Science and Technology Press, 2006. 116-118

16 Liu Y Q, Zhang Y Z, Gao P J. Novel concentration-killing curve method for estimation of bactericidal potency of antibiotics in an in vitro dynamic model. Antimicrob Agents Chemother, 2004, 48: 3884-3891

17 Wang S J, Shen X Z, Zong J. Spectrofluormetric determination of norfloxacin. Chin Medi Pharm, 1993, 24: 411-413

18 Bagel S, Hullen V, Wiedemann B, et al. Impact of gyrA and parC mutations on quinolone resistance, doubling time, and supercoiling degree of Escherichia coli. Antimicrob Agents Chemother, 1999, 43: $868-875$

19 Liu Y Q, Zhang H Q, Hu M, et al. The methodological limitation of antibiotic susceptibility test and its improvement. J Shandong University (Health Science), 2011, 49: 124-132

20 Brown D F. Determination of MICs by the E test. J Antimicrob Chemother, 1992, 29: 455

21 Hill G B, Schalkowsky S. Development and evaluation of the spiral gradient endpoint method for susceptibility testing of anaerobic gram-negative bacilli. Rev Infect Dis, 1990, 12: S200-S209

22 Cooper K E. Theory of antibiotic inhibition zones in agar media. Nature, 1995, 175: 510-511

23 Finn R K. Theory of agar diffusion methods for bioassay. Anal Chem, 1959, 31: 975-977

24 Awerbuch T E, Lustman L. A mathematical model for determining minimum inhibitory concentration (MIC) via diffusion assay. J Theory Biol, 1987, 129: 219-230

25 Ringertz S, Kronvall G. On the theory of the disk diffusion test. APMIS, 1988, 96: 484-490

26 Drugeon H B, Juvin M E, Caillon J, et al. Assessment of formulas for calculating critical concentration by the agar diffusion method. Antimicrob Agents Chemother, 1987, 31: 870-875

27 Anderson B. Improved susceptibility disk assay method employing an agar overlay technique. Antimicrob Agents Chemother, 1978, 14: 761-764

28 Bonev B, Hooper J, Parisot J. Principles of assessing bacterial susceptibility to antibiotics using the agar diffusion method. J Antimicrob Chemother, 2008, 61: 1295-1301

29 Andrews J M. The development of the BASA standardized method of disk diffusion testing. Antimicrob Agent Chemoth, 2001, 48: 2942

30 Turnidge J, Paterson D L. Setting and revising antibacterial susceptibility breakpoints. Clin Microbiol Rev, 2007, 20: 391-408

31 Chen X S. Disk susceptibility test-the limitation and some additional method. Chin J Clin Lab Sci, 1994, 12: 2-5

32 Jorgensen J H, Crawford S A, Fulcher L C, et al. Multilaboratory evaluation of disk diffusion antimicrobial susceptibility testing of Neisseria meningitidis isolates. J Clin Microb, 2006, 44: 17441754

33 Zhao X, Drlica K. Restricting the selection of antibiotic-resistant mutants: measurement and potential uses of the mutant selection window. J Infect Dis, 2002, 185: 561-565

34 Zhang H Q, Liu Y Q, Liu B, et al. A novel approach for estimating growth phase and parameters of bacterial population in batch culture. Sci China Ser C-Life Sci, 2006, 49: 130-140 
35 Liu Y Q, Zhang H Q, Shen J Z, et al. Effect of physiological heterogeneity of $E$. coli population on antibiotic susceptibility test. Sci China Ser C-Life Sci, 2007, 50: 808-813

36 Zhang $\mathrm{H}$ Q, Zhao Y, He X L, et al. A novel approach for assessing the susceptibility of Escherichia coli to antibiotics. Sci China Life Sci, 2010, 53: 1346-1355

37 Cooper K E, Linton A H, Sehgal S N. The effect of inoculum size on inhibition zones in agar media using staphylococci and streptomycin.
J Gen Microbiol, 1958, 18: 670-687

38 Firsov A A, Vostrov S N, Kononenko O V, et al. Prediction of the effects of inoculum size on the antimicrobial action of trovafloxacin and ciprofloxacin against Staphylococcus aureus and Escherichia coli in an in vitro dynamic model. Antimicrob Agents Chemoth, 1999, 43: 498-502

39 Yang J K, Qi X L, Chen L. Biomathematics Overview. Beijing: Science Press, 1982. 640-722

Open Access This article is distributed under the terms of the Creative Commons Attribution License which permits any use, distribution, and reproduction in any medium, provided the original author(s) and source are credited. 